\title{
Spectroscopy and new particles
}

\author{
Tadeusz Lesiak ${ }^{1}$ \\ Institute of Nuclear Physics PAN \\ Radzikowskiego 152, 31-142 Kraków, Poland
}

\begin{abstract}
The properties of three new particles: X(3872), Y(3940) and Z(3931), recently discovered by the Belle collaboration, are briefly reviewed. Negative results of the search for the pentaquark $\Theta(1540)^{+}$are also presented.
\end{abstract}

\section{INTRODUCTION}

In the last two years the Belle collaboration has provided evidence for several new hadrons. This paper presents the observation of three new particles: X(3872), Y(3940) and Z(3931). The other new states discovered recently by Belle were also discussed at this conference in separate talks [1, 2]. The paper also presents the results of searches for the pentaquark state $\Theta(1540)^{+}$. The Belle detector at the KEKB asymmetric $e^{+} e^{-}$ collider [3] is a general purpose spectrometer, described in detail in [4].

\section{PROPERTIES OF THE X(3872)}

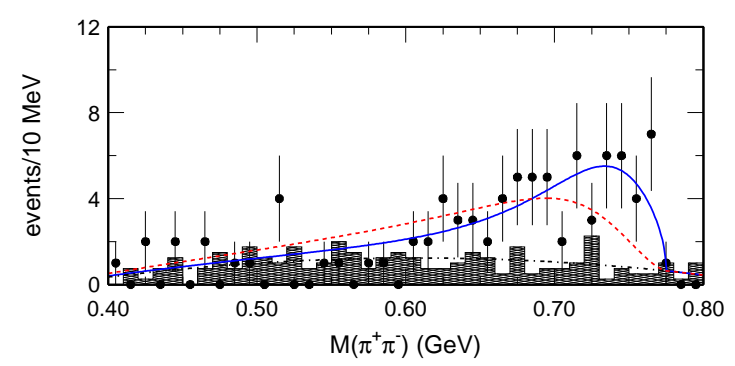

FIGURE 1. The distribution of $\pi^{+} \pi^{-}$invariant mass for events in the $X(3872) \rightarrow \pi^{+} \pi^{-} J / \psi$ signal region (data points). The shaded histogram corresponds to the background as determined by the X-mass sidebands. The solid (dashed) curve denotes a fit that uses a $\rho$ Breit-Wigner shape with the $\rho$ and $J / \psi$ in a relative $S(P)$-wave. The dash-dotted curve shows a smooth parametrization of the background that is used in the fit.

The state X(3872) was discovered by the Belle collaboration in 2003 [5] by analyzing exclusive decays $B^{+} \rightarrow \pi^{+} \pi^{-} J / \psi K^{+}$(charge conjugate modes are included everywhere, unless otherwise specified). The $B$ mesons were reconstructed using two kine-

1 partially supported by the KBN grant No. 2P03B 01324 
matical variables: the energy offset $\Delta E=\sum_{i} E_{i}-E_{\text {beam }}$ and the beam-constrained mass $M_{b c}=\sqrt{E_{\text {beam }}^{2}-\sum_{i}\left(\vec{p}_{i}\right)^{2}}$, where $E_{i}$ and $\vec{p}_{i}$ are the center-of-mass (CMS) energies and momenta of the selected $B$ meson decay products and $E_{\text {beam }}$ is the CMS beam energy. A very narrow peak in the invariant mass spectrum of the system $\pi^{+} \pi^{-} J / \psi$ was observed with a mass of $3872.0 \pm 0.6 \pm 0.5 \mathrm{MeV} / \mathrm{c}^{2}$ and a width below $2.3 \mathrm{MeV}$ (90\% C.L.).

The observation of X(3872) was very quickly confirmed by the CDF [6], D0 [7] and BaBar [8] experiments. The observed decay mode $X \rightarrow \pi^{+} \pi^{-} J / \psi$ seemed to favour the explanation of the $X(3872)$ as an excited charmonium state [9, 10]. However, its properties, in particular the very narrow width, did not allow the identification of the $\mathrm{X}(3872)$ with any $c \bar{c}$ state. At the same time the coincidence of the $\mathrm{X}$ mass with the $D^{0} D^{* 0}$ threshold $\left.(3871.3 \pm 1.0) \mathrm{MeV} / \mathrm{c}^{2}\right)$ has prompted many theoretical speculations that X(3872) may be a so-called deuson i.e. a loosely bound molecular state of these two mesons [11, 12]. Moreover, the $\pi^{+} \pi^{-}$invariant mass distribution (Fig. 11) was found to peak close to the upper kinematical limit of $M\left(\pi^{+} \pi^{-}\right)$as expected for pion-pairs originating from $\rho \rightarrow \pi^{+} \pi^{-}$decays.

Recently, the Belle collaboration, using the $253 \mathrm{fb}^{-1}$ data sample collected at the $\Upsilon(4 S)$ resonance, has provided the first evidence for two new decay modes: $X \rightarrow \gamma J / \psi$ and $X \rightarrow \pi^{+} \pi^{-} \pi^{0} J / \psi[13]$. They were observed in exclusive $B$ meson decays to the final states $\gamma J / \psi K$ and $\pi^{+} \pi^{-} \pi^{0} J / \psi K$, respectively. The yield of the decay $B \rightarrow \gamma J / \psi K$ plotted in bins of the $\gamma J / \psi$ invariant mass (Fig. 2a)) exhibits an excess of $13.6 \pm$ 4.4 events (statistical significance of $4 \sigma$ ). The observation of this decay establishes unambiguously that the charge-conjugation parity of the $\mathrm{X}(3872)$ is positive. The partial width ratio $\Gamma(X \rightarrow \gamma J / \psi) / \Gamma\left(X \rightarrow \pi^{+} \pi^{-} J / \psi\right)$ amounts to $0.14 \pm 0.05$. This result is in contradiction with the $\chi_{c 1}^{\prime}\left(1^{++}\right.$charmonium) assignment for $\mathrm{X}$ as in this case a value around 40 would be expected. The second decay mode $X \rightarrow \pi^{+} \pi^{-} \pi^{0} J / \psi$ was found to be dominated by the sub-threshold decay $X \rightarrow \omega^{*} J / \psi$. This is motivated by the fact that the yield of $B$ mesons plotted in bins of the $\pi^{+} \pi^{-} \pi^{0}$ invariant mass (Fig $2 \mathbf{b}$ )) inside of the signal region from the decay $X \rightarrow \pi^{+} \pi^{-} \pi^{0} J / \psi$ is consistent with zero except for the $M\left(\pi^{+} \pi^{-} \pi^{0}\right)>750 \mathrm{MeV} / \mathrm{c}^{2}$. There, the excess of $12.4 \pm 4.1$ events $(4.3 \sigma)$ is observed. The ratio of branching fractions $\mathrm{B}\left(X \rightarrow \pi^{+} \pi^{-} \pi^{0} J / \psi\right) / \mathrm{B}\left(X \rightarrow \pi^{+} \pi^{-} J / \psi\right)$. was measured to be $1.0 \pm 0.4 \pm 0.3$, which implies a large violation of isospin symmetry.
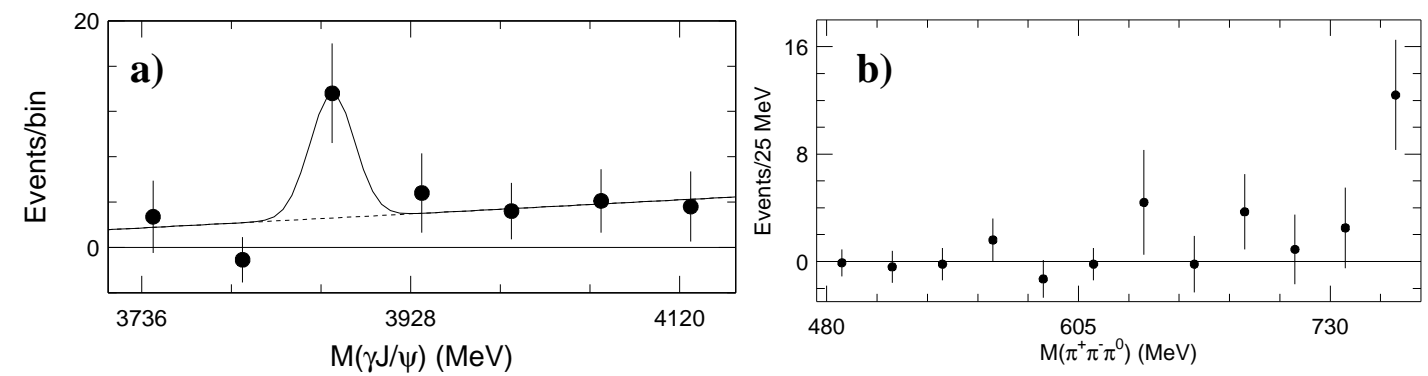

FIGURE 2. The yield of $B$ mesons from the decay a) $B^{0} \rightarrow \gamma J / \psi K$, in bins of the $\gamma J / \psi$ invariant mass and b) $B^{0} \rightarrow \pi^{+} \pi^{-} \pi^{0} J / \psi K$, in bins of the $\pi^{+} \pi^{-} \pi^{0}$ invariant mass, determined from fits to the $\Delta E$ and $M_{b c}$ distributions. 
The Belle collaboration also attempted to determine the $J^{P C}$ quantum numbers of the $\mathrm{X}(3872)$ [14] by studying the angular distributions of the decay $X \rightarrow \pi^{+} \pi^{-} J / \psi$, as suggested by Rosner [15]. Among the twelve possible $J^{P C}$ assignments, half $\left(0^{--}, 0^{+-}\right.$, $1^{--}, 1^{+-}, 2^{--}$and $2^{+-}$) may be discarded due to their negative charge conjugationparity. The value $1^{++}$is in agreement with the data while the assignments $0^{-+}$and $0^{++}$ are strongly disfavoured by the analysis of angular distributions [14]. The additional two odd-parity possibilities: $1^{-+}$and $2^{-+}$are discarded as for them the dipion invariant mass spectrum (Fig. 1) is expected to be much softer to compare with the data. On the other side, the distribution of $M\left(\pi^{+} \pi^{+}\right)$is in agreement with the $1^{++}$hypothesis. The assignment $2^{++}$was strongly disfavoured by the recent, preliminary observation by Belle [16] of the decay $B \rightarrow K X, X \rightarrow D^{0} \overline{D^{0}} \pi^{0}$, where the $11.3 \pm 3.6$ signal events $(5.6 \sigma)$ concentrate close to the threshold for the final state $D^{0} \overline{D^{0}} \pi^{0}$. In the $2^{++}$case, the decay of a spin 2 state to three pseudoscalars $\left(D^{0} \overline{D^{0}} \pi^{0}\right)$ would require at least one pair of them to be in a relative $\mathrm{D}$ wave. In such a configuration the near threshold production would be strongly suppressed by a centrifugal barrier.

All the above observations strongly favour the assignment of $J^{P C}=1^{++}$to the $\mathrm{X}(3872)$. This matches the expectations of models [11, 12] interpreting the $\mathrm{X}$ as a $D^{0} \overline{D^{* 0}}$ bound state. This hypothesis also explains the narrow width of the $\mathrm{X}(3872)$ and the shape of $\pi^{+} \pi^{-}$and $\pi^{+} \pi^{-} \pi^{0}$ spectra in its corresponding decays and leads to the prediction of large isospin violation in the $X$ decays. Among alternative interpretations are: a 'conventional' charmonium [9, 10, 17], glueball [18], tetraquark [19] or the so called cusp effect [20].

\section{EVIDENCE FOR THE Y(3940)}

In 2004 The Belle collaboration provided evidence for another new state, Y(3940), decaying to $\omega J / \psi[21]$. It was again observed in the $B^{+}$meson exclusive decay to the final state $K^{+} \pi^{+} \pi^{-} \pi^{0} J / \psi$. A fit to the $\omega J / \psi$ invariant-mass distribution (Fig 3] yielded a signal of $58 \pm 11$ events $(8.1 \sigma)$ corresponding to a mass of $3943 \pm 11 \pm 13$ $\mathrm{MeV} / \mathrm{c}^{2}$ and the width $87 \pm 22 \pm 26 \mathrm{MeV}$. The $\mathrm{Y}$ mass coincides with that of another

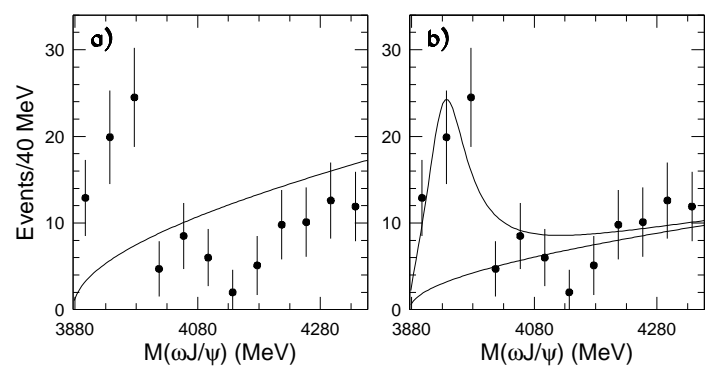

FIGURE 3. $\quad B^{+} \rightarrow K^{+} \omega J / \psi$ signal yields vs $M(\omega J / \psi)$. The curve in (a) shows the result of a fit that includes only a phase-space-like threshold function. The curve in (b) corresponds to the result of a fit that includes an $S$-wave Breit Wigner resonance term. 


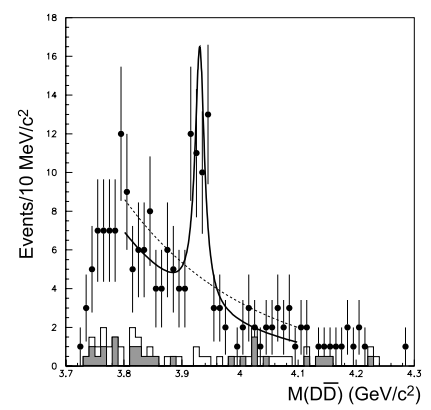

FIGURE 4. Invariant mass distribution of $D \bar{D}$ pairs. The solid (dashed) curve shows the fits with (without) a resonance component. The histograms correspond to the distribution of the events from the $D$-mass sidebands.

particle, X(3940), also observed by Belle [1, 2]. However, it is unlikely that these two states are the same, since the $\mathrm{X}(3940)$ decays to $D \bar{D}^{*}$ and does not decay to $\omega J / \psi$ and the situation is reversed for the Y(3940), as far as the above-mentioned decays are concerned. The properties of $\mathrm{Y}(3940)$ are similar to those expected for the $c \bar{c}-$ gluon hybrid mesons [22].

\section{DISCOVERY OF THE Z(3931)}

A recent search by the Belle collaboration for the production of new resonances in the process $\gamma \gamma \rightarrow D \bar{D}$ [23] yielded evidence for a new state (Fig. 4) at a mass of $3931 \pm 4 \pm 2 \mathrm{MeV} / \mathrm{c}^{2}$ and a width of $20 \pm 8 \pm 3 \mathrm{MeV}$. A signal of $41 \pm 11$ events with a statistical significance of $5.5 \sigma$ was observed. The properties of this new state match the expectations [24, 10] for the radially excited states $\chi_{c 0}^{\prime}$ and $\chi_{c 2}^{\prime}$. A study of angular distributions of the $D$ mesons in the $\gamma \gamma$ rest frame revealed that the data significantly prefer a spin two assignment over spin zero.

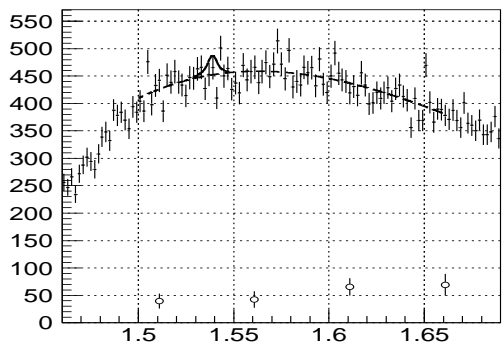

FIGURE 5. Invariant mass spectrum for secondary $p K_{s}^{0}$ pairs and expected yield of the charge exchange reaction per $2 \mathrm{MeV} / \mathrm{c}^{2}$ (open dots). A fit to a third order polynomial is represented by the dashed curve. The $\Theta(1540)^{+}$contribution expected from the DIANA result [26] is presented with the solid line. 


\section{SEARCH FOR THE $\Theta(1540)^{+}$}

The Belle collaboration has searched for both inclusive and exclusive production of the $\Theta(1540)^{+}$pentaquark using kaon secondary interactions in the material of the detector [25]. An upper limit of $2.5 \%$ (90\% C.L.) was set on the ratio of the $\Theta(1540)^{+}$ to $\Lambda(1520)$ inclusive production cross section. The search for the exclusive production of the $\Theta(1540)^{+}$as an intermediate resonance in the charge exchange reaction $K^{+} n \rightarrow p K_{s}^{0}$

yielded an upper limit of $\Gamma_{\Theta^{+}}<0.64 \mathrm{MeV}\left(90 \%\right.$ C.L.) at $m_{\Theta^{+}}=1.539 \mathrm{MeV} / \mathrm{c}^{2}$. This value is below the current Particle Data Group [27] value of $0.9 \pm 0.3$.

\section{SUMMARY}

The properties of three new particles: X(3872), Y(3940) and Z(3931), recently observed by the Belle collaboration,, were reviewed. For the $\mathrm{X}(3872)$ the observation of new decay modes together with angular analysis of the $\pi^{+} \pi^{-} J / \psi$ favours the assignment $J^{P C}=$ $1^{++}$and is in agreement with the deuson hypothesis. The most plausible interpretations of the $\mathrm{Y}(3940)$ and $\mathrm{Z}(3931)$ are the $c \bar{c}-$ gluon and $\chi_{c 2}^{\prime}$, respectively. The search for the $\Theta(1540)^{+}$yielded a null result giving rise to the limit $\Gamma_{\Theta^{+}}<0.64 \mathrm{MeV}$ (90\% C.L.).

\section{REFERENCES}

1. S. Korpar, these proceedings.

2. K. Trabelsi, these proceedings.

3. S. Kurokawa, E. Kikutani, Nucl. Instrum. Methods A, 499, 1 (2003), and other papers incl. in this vol.

4. Belle Collaboration, A. Abashian et al., Nucl. Instrum. Methods A, 479, 117 (2002).

5. Belle Collaboration, S.-K. Choi,S. L. Olsen et al., Phys. Rev. Lett., 91, 262001 (2003).

6. CDF-II Collaboration, D. Acosta et al., Phys. Rev. Lett., 93, 072001 (2004).

7. D0 Collaboration, V. M. Abazov et al., Phys. Rev. Lett., 93, 162002 (2004).

8. BaBar Collaboration, B. Aubert et al., Phys. Rev. D, 71, 071003 (2004).

9. E. J. Eichten, K. Lane and C. Quigg, Phys. Rev. Lett., 89, 162002 (2002).

10. E. J. Eichten, K. Lane and C. Quigg, Phys. Rev. D, 69, 094019 (2004).

11. N. A. Törnqvist, Phys. Lett. B, 590, 209 (2004).

12. E. S. Swanson, Phys. Lett. B, 588, 189 (2004).

13. Belle Collaboration, K. Abe et al., hep-ex/0505037

14. Belle Collaboration, K. Abe et al., hep-ex/0505038

15. J. Rosner, Phys. Rev. D, 70, 094023 (2004).

16. P.Pakhlov, procedings of the HEP2005 International Europhysics Conf. on High Energy Physics.

17. T. Barnes, S. Godfrey, Phys. Rev. D, 69, 054008 (2004).

18. K. K. Seth, Phys. Lett. B, 612, 1 (2005).

19. L. Maiani et al, Phys. Rev. D, 71, 014028 (2005).

20. D.Bugg, Phys. Lett. B, 598, 8 (2004).

21. Belle Collaboration, S.-K. Choi,S. L. Olsen et al., Phys. Rev. Lett., 94, 182002 (2005).

22. F. E. Close, I. Dunietz et al., Phys. Rev. D, 57, 5653 (1998);

G. Chiladze, A.F. Falk, A. A. Petrov, Phys. Rev. D, 58, 034013 (1998);

F. E. Close and S. Godfrey, Phys. Lett. B., 574, 210 (2003).

23. Belle Collaboration, K. Abe et al., hep-ex/0507033

24. S. Godfrey and N. Isgur, Phys. Rev. D., 32, 189 (1985).

25. Belle Collaboration, K. Abe et al., hep-ex/0507014 subm to Phys. Lett. B.

26. DIANA Collaboration, V. Barmin et al., Phys. Atom. Nucl., 66, 1715 (2003). 
27. S. Eidelman et al., Phys. Lett. B, 592, 1 (2004). 


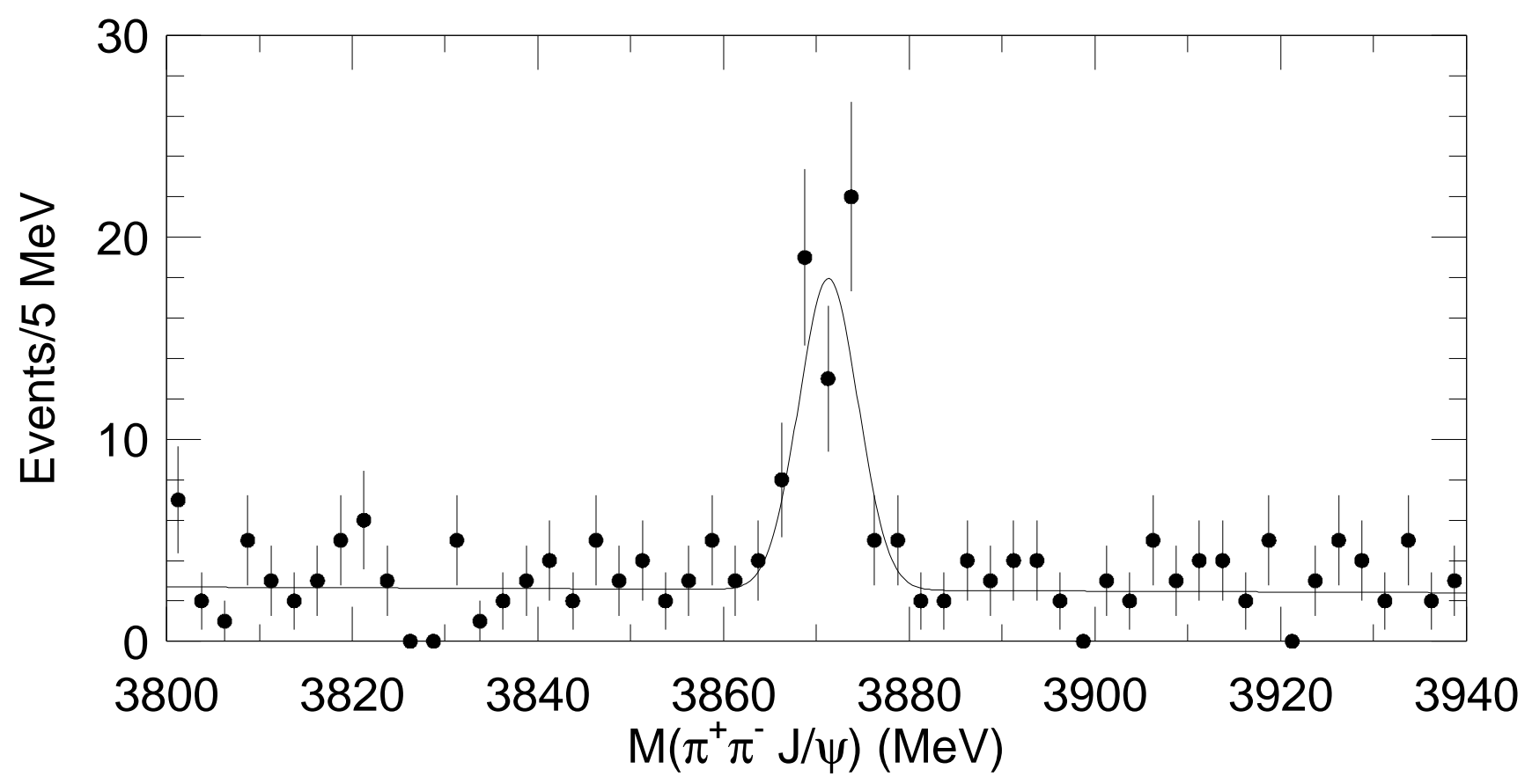



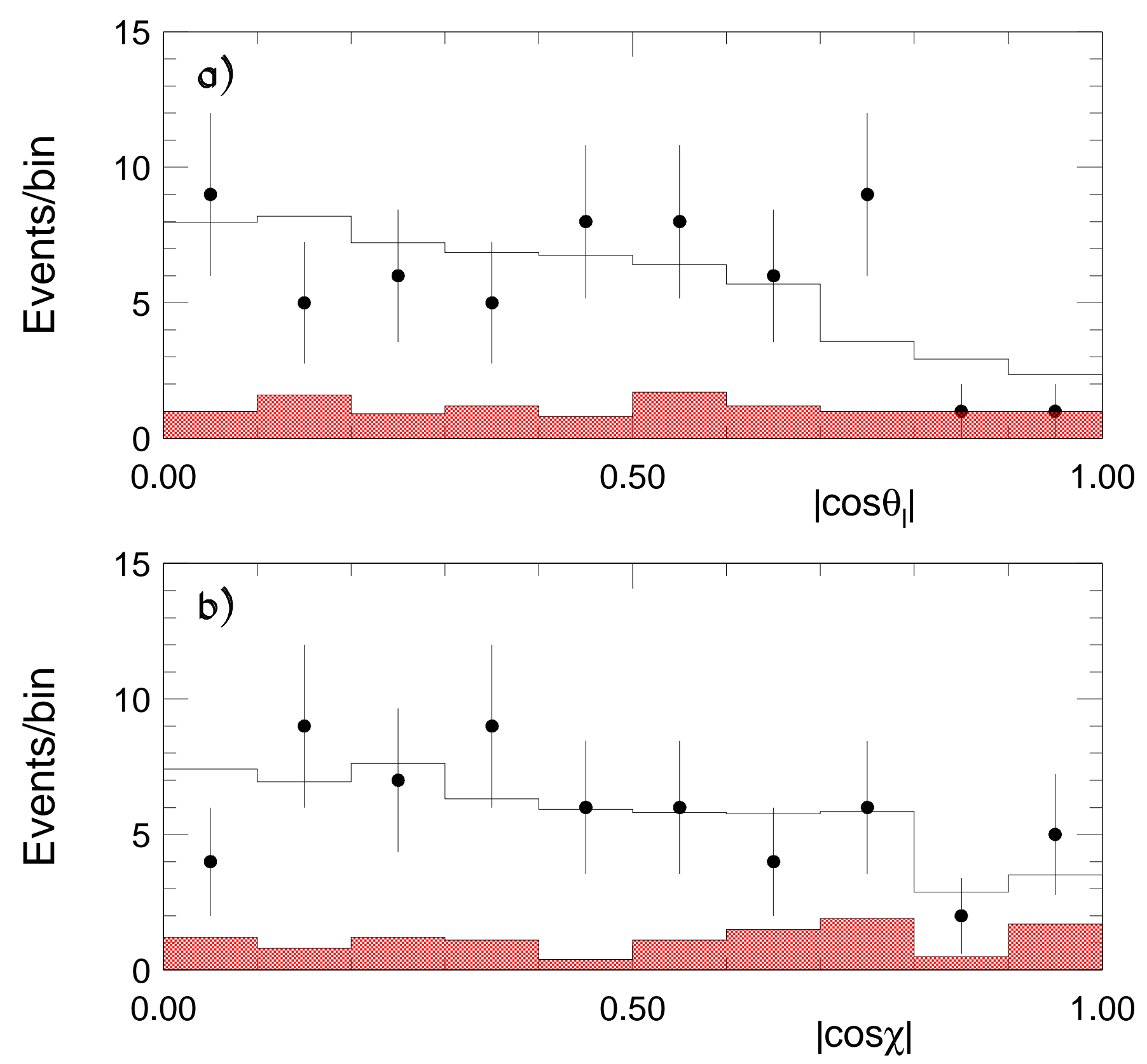


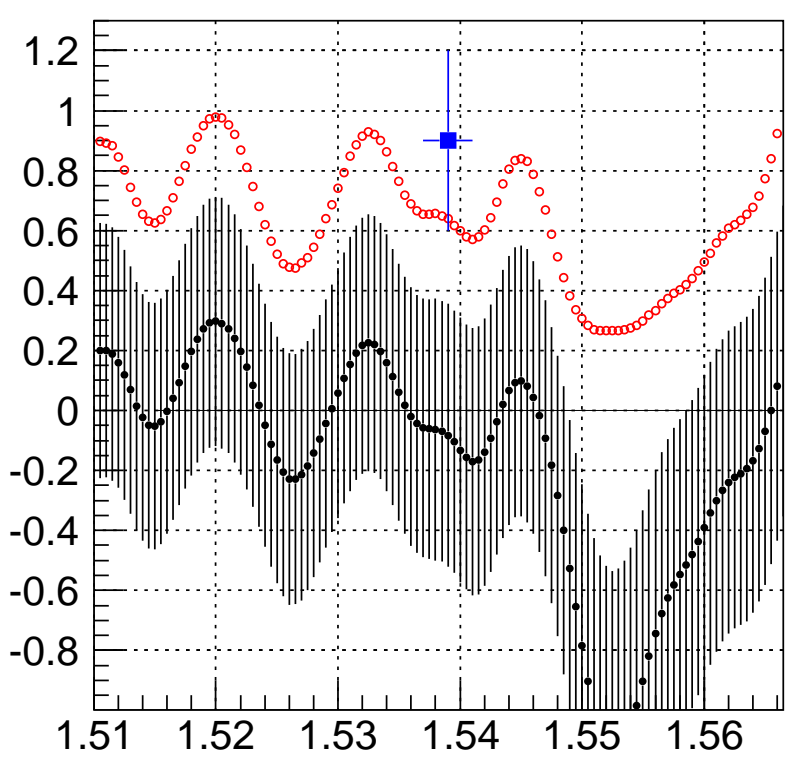


